\title{
Study of ortho-para effects on muonic-atomic and muonic-molecular processes in $\mathbf{d d}-\mu \mathrm{CF}$
}

\author{
H. Imao ${ }^{a b}$, N. Kawamura ${ }^{a}$, K. Ishida $^{c}$, T. Matsuzaki ${ }^{c}$, \\ Y. Matsuda ${ }^{c}$, A. Toyoda ${ }^{a}$ and K. Nagamine ${ }^{a d}$ \\ $a_{\text {KEK-MSL, Tsukuba, Ibaraki, Japan }}$ \\ $b$ University of Tokyo, Bunkyo-ku, Tokyo, Japan \\ c RIKEN, Wako, Saitama, Japan \\ $d$ University of California, Riverside, USA
}

\begin{abstract}
The effect of ortho-para composition of hydrogen isotope molecule on muoncatalyzed fusion $(\mu \mathrm{CF})$ phenomenon in solid, liquid, and gaseous deuterium has been clearly observed. A drastic difference between the ortho-para effect in liquid phase and that in gas phase has been discovered. The ortho-para effect only in gas has been qualitatively consistent with the theoretical prediction without the condensed matter effect.
\end{abstract}

\section{Introduction}

The $\mu \mathrm{CF}$ phenomena were extensively investigated in a wide range of temperatures, phases and densities with various combinations of hydrogen-isotope after the discovery of the resonant mechanism of $d d \mu$ molecular formation [1]. The studies of $\mu \mathrm{CF}$ in pure deuterium (dd- $\mu \mathrm{CF}$ ), especially, have played an essential role to understand the basic mechanisms of $\mu \mathrm{CF}$. In gas phase, the excellent agreement has been achieved between the experimental value of the $d d \mu$ resonant molecular formation rate $\tilde{\lambda}_{\frac{3}{2}}$ and that of the conventional $\mu \mathrm{CF}$ theory, which assumes that the epithermal $\mathrm{d} \mu$ is rapidly thermalized and $\mathrm{D}_{2}$ is not interacting with its neighbors $[2,3]$. In solid, however, unexpected high resonant molecular formation rates have been observed in several experiments $[4,5,6]$. This has been a longstanding problem in dd- $\mu \mathrm{CF}$. In order to understand the nature of the solid-state effect in $\mu \mathrm{CF}$, the ortho-para effect on dd- $\mu \mathrm{CF}$ were measured with a thin solid deuterium layer target at a temperature of $3.5 \mathrm{~K}$ [6]. The result showed the opposite tendency against 
the theory for dilute gas, which predicted the enhancement of these rates in ortho deuterium $[7,8]$. Recently, the advanced theoretical study for $\mu \mathrm{CF}$ processes in condensed deuterium has been developed by Adamczak $[9,10]$. The theory proposed by Adamczak was able to explain the high resonant molecular formation rates in solid. However, the theoretical ortho-para effect on the proton time spectrum, which was derived from the Monte-Carlo simulation on the $\mathrm{d} \mu$ scattering and the molecular formation in solid deuterium [10], were not consistent with that observed by Toyoda [6]. In order to obtain a comprehensive understanding of the nature of the condensed-matter effect and ortho-para dependent phenomena in $\mathrm{dd}-\mu \mathrm{CF}$, we observed the dependence of $\mathrm{dd}-\mu \mathrm{CF}$ phenomena on the ortho-para states in solid, liquid, and gaseous deuterium.

\section{Experimental}

Our experiments were performed at TRIUMF M9B muon channel using a backwards decay $\mu^{-}$beam of $52 \mathrm{MeV} / \mathrm{c}$ momentum. A schematic figure of our experimental setup is shown in Fig. 1. A muon incoming signal was generated by the coincidence of the signals
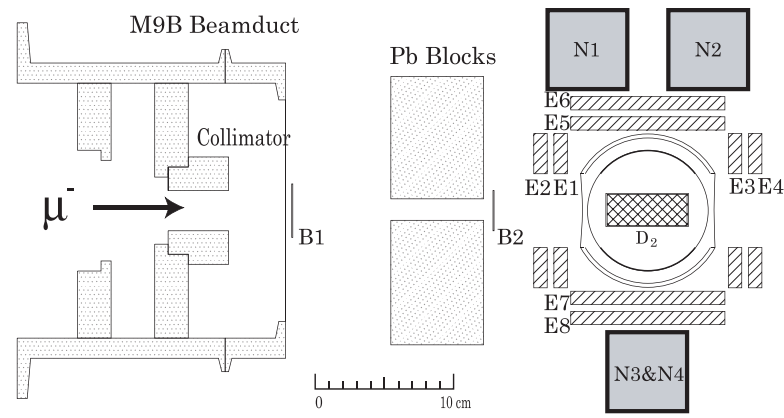

Figure 1: Schematic view of the experimental setup for d-d fusion neutron measurements with solid or liquid ortho-para controlled deuterium.

of two beamline counters $(\mathrm{B} 1 \times \mathrm{B} 2)$, and the signal opened a $12-\mu$ s gate for data taking. As a neutron detector for detecting $2.5-\mathrm{MeV}$ fusion neutrons, four NE-213 liquid scintillators (N1-N4) were placed perpendicular to the incoming $\mu^{-}$beam axis. NE-213 was chosen for its pulse shape discrimination capability of $n-\gamma$. Muon-decay electrons were detected by four pairs of plastic scintillation counters (E1-E8) placed around the target. The target cell for solid or liquid state is a cylindrical oxygen free high conductivity (OFHC) copper container $(\phi 30 \mathrm{~mm} \times \mathrm{L} 30 \mathrm{~mm})$. For the gas target at $36.2 \mathrm{~K}$, we used 240 -cc OFHC copper target cell, which was sufficient to sustain the maximum operating pressure of $12 \mathrm{bar}$. Pd filter was used for purification of deuterium. The ortho-rich deuterium was prepared by employing a paramagnetic conversion method [11]. In order to determine the ortho-para composition of deuterium, a rotational Raman spectroscopy method was employed for the first time in $\mu \mathrm{CF}$. The Raman laser system consisted of a Diode Pumped Solid State 
(DPSS) blue laser, optic system for the laser inducing and the scattered light collecting, a sample cell, and the single grating spectrometer connected with a photo counting system. The DPSS laser outputs 20mW-CW. The Raman measurement was carried out before each exposure run to check that our expected ortho-para composition was achieved. The measurement was also carried out after the run to check that the ortho-para composition was unchanged by muon irradiation. The ortho-para composition was determined within $2 \%$ error. The main observable quantity in our experiment was the $2.5-\mathrm{MeV}$ fusion neutron emitted from d-d fusion reaction. We measured and analyzed time distribution of the fusion neutrons from both ortho and normal target in the temperature range from 5.5 to $36.2 \mathrm{~K}$. The main background came from the capture neutrons emitted from the muon nuclear capture reactions in the surrounding material. The background signal by these capture neutrons were strongly suppressed by demanding that an electron was detected between $0.3 \mu \mathrm{s}$ and $5 \mu \mathrm{s}$ after the neutron event. Gaseous hydrogen target were used to estimate the remaining background. The time spectrum of $\mathrm{d}$ - $\mathrm{d}$ fusion neutrons after background subtraction was fitted with the expected function derived from the general kinetics model for dd- $\mu \mathrm{CF}[12]$. All observed spectra were normalized based on the detected number of $\mu$-e decay electrons.

\section{Results and disscussion}

Table 1 shows our results of the resonant molecular formation rate $\tilde{\lambda}_{\frac{3}{2}}$, the hyperfine transition rate $\tilde{\lambda}_{\frac{3}{2}} \frac{1}{2}$, and the non-resonant molecular formation rate $\tilde{\lambda}_{\frac{1}{2}}{ }^{2}$ for normal- and ortho-deuterium, respectively. All of the resulting values for the $\tilde{\lambda}_{\frac{3}{2}}$ and $\tilde{\lambda}_{\frac{3}{2} \frac{1}{2}}$ in normal deuterium have agreed with those in the previous experiments $[4,5,6]$. In solid and liquid phases, the decrease in the rates of $\tilde{\lambda}_{\frac{3}{2}}$ and $\tilde{\lambda}_{\frac{3}{2} \frac{1}{2}}$ for ortho deuterium was observed. This effect is opposite to that predicted by the conventional theory for gas $[7,8]$. The ortho-para effect in solid phase has been consistent with that in the previous experiment [6]. The rates of $\tilde{\lambda}_{\frac{1}{2}}$ were independent of the ortho-para state of deuterium within experimental error. It is worth noting that the decrease of $\tilde{\lambda}_{\frac{3}{2}}$ and $\tilde{\lambda}_{\frac{3}{2}} \frac{1}{2}$ in ortho was observed even in liquid phase. The lower resonant molecular formation rate in ortho deuterium at $23.2 \mathrm{~K}$ than that in normal deuterium cannot be explained by the non-thermalization effect of $\mathrm{d} \mu$ in normal deuterium, because the experimental rates of $\tilde{\lambda}_{\frac{3}{2}}$ in liquid deuterium are not higher than those of the conventional theory, which assumes the rapid thermalization of $\mathrm{d} \mu$. Thus, it is probable that the resonant conditions in solid and liquid phases are different from the theoretical resonant condition in gas phase. In addition, such a changing of the resonant condition should be caused by the condensed-matter effect, which is common to liquid and solid deuterium rather than an effect specific to solid only. The measurement of fusion neutron yields in normal- and ortho-deuterium was also carried out for gaseous state at $36.2 \mathrm{~K}$. For the ortho deuterium, unexpected delayed build-up structure was observed (the inset of Fig. 2) overlapping the exponential decay time spectrum in the time region before $100 \mathrm{~ns}$ while such a structure was not evident in any other target conditions. Although the time spectrum for ortho deuterium gas could not be completely explained within the conventional static $\mu \mathrm{CF}$ theory, one possible explanation for the time structure is slow 
Table 1: The results of the ortho-para effect on $\tilde{\lambda}_{\frac{3}{2}}, \tilde{\lambda}_{\frac{3}{2} \frac{1}{2}}$, and $\tilde{\lambda}_{\frac{1}{2}}$ are listed. The density is in units of LHD (liquid hydrogen density).

\begin{tabular}{|c|c|c|c|c|c|c|}
\hline $\begin{array}{c}\text { Temp. } \\
{[\mathrm{K}]}\end{array}$ & $\begin{array}{c}\text { Density } \\
{[\text { LHD] }}\end{array}$ & State & $\begin{array}{c}\text { Ortho } \\
{[\%]}\end{array}$ & $\begin{array}{c}\lambda_{\frac{3}{2}} \\
{\left[\mu \mathrm{s}^{-1}\right]}\end{array}$ & $\begin{array}{c}\lambda_{\frac{3}{2} \frac{1}{2}} \\
{\left[\mu \mathrm{s}^{-1}\right]}\end{array}$ & $\begin{array}{c}\lambda_{\frac{1}{2}} \\
{\left[\mu \mathrm{s}^{-1}\right]}\end{array}$ \\
\hline \multirow[t]{2}{*}{5.5} & 1.43 & solid & $67(2)$ & $2.35(5)$ & $31.6(4)$ & 0.044 (fix) \\
\hline & & & $99(1)$ & $1.98(5)$ & $28.3(4)$ & $0.043(2)$ \\
\hline \multirow[t]{2}{*}{11.7} & 1.42 & solid & $67(2)$ & $2.61(9)$ & $32.0(8)$ & $0.040(3)$ \\
\hline & & & $99(1)$ & $2.15(8)$ & $30.1(8)$ & $0.041(3)$ \\
\hline \multirow[t]{2}{*}{18.2} & 1.40 & solid & $67(2)$ & $2.46(5)$ & $33.2(4)$ & $0.043(2)$ \\
\hline & & & $99(1)$ & $2.11(5)$ & $30.8(4)$ & $0.045(2)$ \\
\hline \multirow[t]{2}{*}{18.8} & 1.23 & liquid & $67(2)$ & $2.70(6)$ & $32.4(4)$ & $0.044(2)$ \\
\hline & & & $99(1)$ & $2.32(5)$ & $30.2(4)$ & $0.045(2)$ \\
\hline \multirow[t]{2}{*}{21.2} & 1.19 & liquid & $67(2)$ & $2.91(8)$ & $33.0(6)$ & $0.048(3)$ \\
\hline & & & $99(1)$ & $2.34(7)$ & $29.1(6)$ & $0.046(3)$ \\
\hline \multirow[t]{2}{*}{23.2} & 1.16 & liquid & $67(2)$ & $2.86(6)$ & $32.6(4)$ & $0.047(2)$ \\
\hline & & & $99(1)$ & $2.38(5)$ & $29.8(4)$ & $0.048(2)$ \\
\hline \multirow[t]{2}{*}{36.2} & 0.17 & gas & $67(2)$ & $4.59(10)$ & $40.5(4)$ & 0.049 (fix) \\
\hline & & & $99(1)$ & - & - & $0.050(2)$ \\
\hline
\end{tabular}

completion of thermalization of $\mathrm{d} \mu$ atoms. Fig. 2 shows the temperature dependence of the number of resonant fusion neutrons per muon in normal and ortho deuterium. Theories for the ortho-para effect based on gas model [7,8], which predicted enhanced resonant fusion yield in ortho deuterium, is consistent with our result for gaseous deuterium only and is not enough to explain our result for liquid and solid phases even qualitatively.

\section{Conclusion}

The effect of ortho-para composition on $\mu \mathrm{CF}$ in solid, liquid, and gaseous deuterium has been clearly observed. The decrease in the rate of $\tilde{\lambda}_{\frac{3}{2}}$ and $\tilde{\lambda}_{\frac{3}{2}} \frac{1}{2}$ for ortho-rich deuterium was observed not only in solid phase but also in liquid phase. On the other hand, in gas phase, the fusion neutron yield has been significantly enhanced in ortho-rich deuterium. The ortho-para effect to fusion neutron yield in gas is opposite to that in solid and liquid. A precise theoretical study of our experimental result taking account of condensed matter effects such as the shifts of resonant conditions is expected to provide the deeper understanding of $\mu \mathrm{CF}$ and is strongly waited for.

\section{Acknowledgment}

Financial support in past from the Japan Society for the Promotion of Science is acknowledged. Helpful discussion with Profs. L.I. Ponomarev, M.P. Faifmand and A. Adamczak are also acknowledged. 


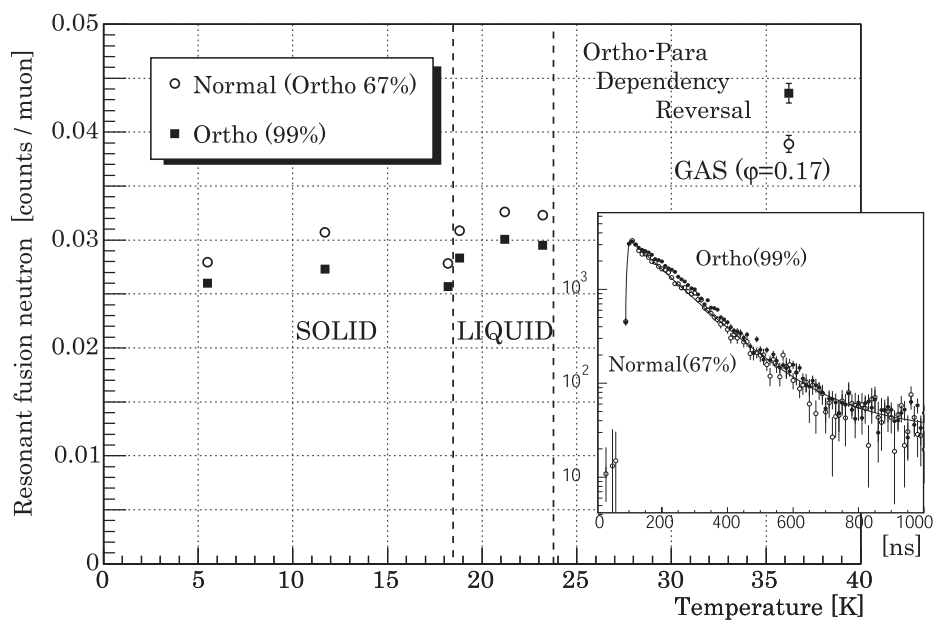

Figure 2: The temperature dependence of resonant fusion neutron yield per muon. The density of gaseous deuterium at $36.2 \mathrm{~K}$ is $0.17 \mathrm{LHD}$. The lower-right inset shows the fusion neutron time spectra with gaseous normal- and ortho-deuterium.

\section{References}

[1] E.A.Vesman, Zh. Eks. Theo. Fiz. Pis. 5 (1967) 50. (Sov. Phys. JETP Lett. 591 (1967).)

[2] C. Petitjean et al., Hyperfine Interactions 138, 191 (2001).

[3] M.P. Faifman, L.I. Men'shikov, and T.A. Strizh, Muon Catalyzed Fusion 4, 1 (1989).

[4] P.E. Knowles et al., Phys. ReV. A56, 1970 (1997).

[5] D.L. Demin et al., Hyperfine Interactions 101/102, 13 (1996).

[6] A. Toyoda et al., Phys. Rev. Lett. 90, 243401 (2003).

[7] M. Leon and J.S. Cohen, Phys. Rev. A31 2680 (1985).

[8] M.P. Faifman, private communication.

[9] A. Adamczak and M.P. Faifman, Phys. Rev. A64, 052705 (2001).

[10] A. Adamczak, Habilitation thesis, Henryk Niewodniczański Inst. of Nucl. Phys., (2003).

[11] Isaac F. Silvera, Review of Modern Physics 52, 393 (1980).

[12] A. Scrinzi et al., Phys. Rev. A47, 4691 (1993). 
